\title{
LIST OF REVIEWERS FOR VOLUME 60
}

The editors of Brittonia are grateful to the following and 44 anonymous reviewers for their willing assistance with manuscripts considered for publication in Volume 60.

\author{
Mac Alford \\ Frank Almeda \\ Marccus Alvez \\ Alexander Antonelli \\ C. C. Berg \\ Rodrigo Bernal \\ Mario Blanco \\ Finn Borschenius \\ Michael Boudrie \\ Ken Cameron \\ George Cremers \\ H. Esser \\ Eduardo Estrada \\ Don Ferrar \\ Enrique Forero \\ Reneé Fortunato
}

\author{
Renato Goldenberg \\ Ron Hartman \\ Jackie Kallunki \\ Lucia Kawasaki \\ Lawrence Kelly \\ Tom Lammers \\ Les Landrum \\ Barney Lipscomb \\ Pete Lowry \\ James Macklin \\ Fabian Michelangeli \\ Robbin Moran \\ Geoff Levin \\ Gwilym Lewis \\ James Luteyn \\ Michael Nee
}

\author{
Roy Osborne \\ Monica Ponce \\ G. Queiroz \\ Tom Ranker \\ Harold Robinson \\ Germinal Rouhan \\ Andrew Salywon \\ Mario Sousa Sánchez \\ Alan Smith \\ Michael Sundue \\ Daniel Tejero \\ Carmen Ulloa \\ S. Vogel \\ Tom Zanoni
}

